\title{
CONTRIBUTO ALLA CONOSCENZA DI TAXA ENDEMICI DELLA SICILIA ATTRAVERSO L'ANALISI MORFOBIOMETRICA DEL POLLINE
}

\author{
Walter DE LEONARDIS, Rosina MATARESE PALMIERI, \\ Michelangelo ROSSITTO \& Antonina ZIZZA
}

RIASSUNTO: E' caratterizzato il polline di otto taxa endemici mediante indagine biometrica al microscopio ottico ed analisi dell'ultrastruttura dello sporoderma al microscopio elettronico a scansione ed a trasmissione. I taxa esaminati sono: Trifolium fragiferum L. ssp. bonannii (C. Presl) Sojàk, Laserpitium garganicum (Ten.) Bertol. ssp. siculum (Sprengel) Pign., Senecio ambiguus (Biv.) DC., Senecio aethnensis Jan ex DC. (endemismi della Sicilia), Cerastium tomentosum L., Polygala preslii Sprengel, Pimpinella anisoides Briganti, Senecio lycopifolius Desf. ex Poiret (endemismi dell'Italia Centro-Meridionale e della Sicilia).

Parole chiave: Palinologia, endemismi, Sicilia, Italia Centro-Meridionale.

ABSTRACT: A contribution to the knoledge of endemic taxa from Sicily by a morphobyometric study of the pollen.

The pollen grains of eight endemic taxa have been characterized by a byometric and ultrastructural study with light, scanning electron and trasmission electron microscopy. They are: Trifolium fragiferum L. ssp. bonannii (C. Presl) Sojàk, Laserpitium garganicum (Ten.) Bertol. ssp. siculum (Sprengel) Pign., Senecio ambiguus(Biv.) DC., Senecio aethnensis Jan ex DC. (endemic taxa to Sicily), Cerastium tomentosum L., Polygala preslii Sprengel, Pimpinella anisoides Briganti, Senecio lycopifolius Desf. ex Poiret (endemic taxa to CentralSouthern Italy and Sicily).

Key words: Palynology, endemisms, Sicily, Central-Southern Italy.

\section{INTRODUZIONE}

Scopo di questa ricerca è lo studio biometrico ed ultrastrutturale del polline di otto taxa endemici della Sicilia per il ruolo fondamentale che gli endemismi svolgono nella ricostruzione della storia della vegetazione (Bertolani et al. 1964).

Il contributo, che si collega a precedenti lavori palinologici su entità siciliane (Accorsi et al. 1984; De Leonardis et al. 1984a, 1984b, 1986a), s'inquadra in un programma di studio - gestito da una banca dati palinologica (Aiello et al. 1983, 1984; De Leonardis et al. 1985) - sulla caratterizzazione morfobiometrica dei pollini della Flora Siciliana. 


\section{MATERIALE E METODI}

Tutti i campioni di polline sono stati sottoposti ad acetolisi secondo la tecnica di Erdtman (1960). Sono stati osservati al microscopio Zeiss standard, obiettivo ad immersione 100x (N.A. 1.30) ed oculare complex 10x.

Le osservazioni al microscopio elettronico a trasmissione sono state effettuate al Siemens Elmiskop 102 A. Il polline, per queste osservazioni, dopo l'acetolisi è stato lavato con acqua distillata e centrifugato. Il pellet, disidratato con serie crescenti di alcool, è stato trattato con ossido di propilene e quindi incluso in Epon-Araldite (Mollenhauer 1964). Successivamente le sezioni ultrafini (600 $\AA$ ), ottenute con l'ultramicrotomo LKB $2088 \mathrm{~V}$, sono state colorate con acetato di uranile e citrato di piombo (Reynolds 1963).

Per l'osservazione al microscopio elettronico a scansione il polline acetolizzato è stato lavato e disidratato; successivamente, deposto su filtri millipore, è stato essiccato all'aria e ricoperto da un sottile strato di oro (100 $\AA$ ) mediante evaporatore Edwards S 150A. Le osservazioni sono state effettuate al Jeol Scanning Microscope 35.

E' stata adottata la terminologia di Faegri e Iversen (1975), Hideux e Ferguson (1976), De Leonardis et al. (1986b). I termini struttura e scultura dell'esina sono stati interpretati sensu Praglowski (1975) e Praglowski e Raj (1979).

Abbreviazioni adottate. Apert: apertura; c: columella; ca: cavea; ci: columella intratectale; co: costa; DIM: Dimensione; DM: Diametro maggiore; Dm: Diametro minore; E: Asse equatoriale; en: endexina; fl: foot-layer; l: lumen; m: margo; mp: microperforazione; mpo: membrana poro; Nc: Numero colpi; Np: Numero pori; NPC: Numero, Posizione e Caratteristica delle aperture; P: Asse polare; Perim.: Perimetro; P. es.: Spessore esina polare; Polar: Polarità; RAGG.: Raggruppamento; SIMM.: Simmetria; Spi.h: Altezza spinae; t: tectum.

\section{RIS ULTATI E DISCUSSIONE}

\section{Cerastium tomentosum $\mathrm{L}$.}

RAGG.: monadi - SIMM.: radiosimmetrici - POLAR.: apolari - PERIM.: circolari $47 \%$, subcircolari $50 \%$, ovali $3 \%$ - FORMA: oblato sferoidali * $30 \%$, prolato sferoidali* $67 \%$, subprolati *3\% - NPC: 364 polipantoporati - APERT.: pori con membrana ed operculum - Np: 6-8 - ESINA: tectata microperforata spinulata - DIM.: DM 48(42)37, Dm 44(40)35.

\section{Trifolium fragiferum L. ssp. bonannii (C. Presl) Sojàk}

RAGG.: monadi - SIMM.: radiosimmetrici - POLAR.: isopolari - PERIM.: vis. pol. subtriangolari pticotremi; vis. equat. circolari 3\%, subcircolari 13\%, ovali $80 \%$, ellittici $4 \%$ - FORMA: prolato sferoidali $17 \%$, subprolati $80 \%$, prolati $3 \%$ NPC: 345 trizonocolporati - APERT.: ora lolongati; colpi fusiformi con margo e costae - ESINA: tectata soprareticolata - DIM.: P 28(25)21, E 22(21)18.

Polygala preslii Sprengel

RAGG.: monadi - SIMM.: radiosimmetrici - POLAR.: isopolari - PERIM.: 
vis. pol. subcircolari con poroidi polari; vis. equat. circolari $43 \%$, subcircolari $50 \%$, ovali $7 \%$ - FORMA: oblato sferoidali $87 \%$, prolato sferoidali $7 \%$, suboblati $3 \%$, subprolati 3\% - NPC: 745 polizonocolporati - APERT.: ora zonoporati: colpi a margini retti con apici arrotondati - Nc: 10-13 - ESINA: tectata psilata - DIM.: P 41(36)33, E 41(38)33.

\section{Pimpinella anisoides Briganti}

RAGG.: monadi - SIMM.: radiosimmetrici - POLAR.: isopolari - PERIM.: vis. pol. subtriangolari pticotremi; vis. equat. ellittici - FORMA: prolati 10\%, perprolati 90\%, rettangolari apiculati - NPC: 345 trizonocolporati - APERT.: ora lalongati ; colpi stretti ad apici acuti - ESINA: rugulata con ipertrofia intermedia (tra area polare ed area equatoriale) - DIM.: P 37(33)30, E 17(14)13.

Laserpitium garganicum (Ten.) Bertol. ssp. siculum (Sprengel) Pign.

RAGG.: monadi - SIMM.: radiosimmetrici - POLAR.: isopolari - PERIM.: vis. pol. subtriangolari pticotremi; vis. equat. ellittici, rettangolari apiculati FORMA: prolati 57\%, perprolati 43\% - NPC: 345 trizonocolporati - APERT.: ora lalongati; colpi fusiformi ad apici acuti - ESINA: rugulata con ipertrofia columellare polare - DIM.: P 40(37)31, E 21(18)15.

Senecio lycopifolius Desf. ex Poiret

RAGG.: monadi - SIMM.: radiosimmetrici - POLAR.: isopolari - PERIM.: vis. pol. subtriangolari pticotremi (sincolpati $25 \%$ ); vis. equat. circolari $37 \%$, subcircolari $63 \%$ - FORMA: oblato sferoidali $70 \%$, prolato sferoidali $30 \%$ - NPC: 345 trizonocolporati $75 \%$, trizonocolporoidati $25 \%$ - APERT.: ora lolongati; colpi fusiformi - ESINA: tectata perforata con spinae Spi. h 4(3)2 - DIM.: P 32(29)24, E $31(29) 26$.

Senecio ambiguus (Biv.) DC

RAGG.: monadi - SIMM.: radiosimmetrici - POLAR.: isopolari - PERIM.: vis. pol. subtriangolari pticotremi (sincolpati 20\%); vis. equat. circolari $50 \%$, subcircolari $47 \%$, ovali 3\% - FORMA: prolato sferoidali $20 \%$, oblato sferoidali $77 \%$, suboblati 3\% - NPC: 345 trizonocolporati 75\%, trizonocolporoidati $25 \%$ APERT.: ora lolongati; colpi fusiformi o con bordi sinuosi. Presenza di vestibulum - ESINA: tectata perforata con spinae Spi. h 4(4)3 - DIM.: P 30(27)21, E 30(27)22.

Senecio aethnensis Jan ex Dc.

RAGG.: monadi - SIMM.: radiosimmetrici - POLAR.: isopolari - PERIM.: vis. pol. subtriangolari pticotremi; vis. equat. circolari $69 \%$, subcircolari $23 \%$, ovali $8 \%$ - FORMA: oblato sferoidali $46 \%$, prolato sferoidali $46 \%$, subprolati $8 \%$ - NPC: 345 trizonocolporoidati - APERT.: ora lolongati; colpi fusiformi - ESINA: tectata perforata con spinae Spi. h. 3(2)1 - DIM.: P 25(22)20, E 23(22)19.

\section{CARYOPHYLLACEAE}

Nowicke (1975) evidenzia nelle Caryophyllaceae tre morfotipi pollinici: - con granuli tricolpati; 
- con granuli polipantoporati ed esina con spinulae e tubuli/puncta. Distinto in due subtipi a seconda che i pori siano o meno infossati in depressioni della parete per la presenza o assenza di convessità esiniche mesoporiali;

- con granuli polipantoporati ad esina reticolata.

Cerastium tomentosum rientra nel primo subtipo del secondo tipo (Tav. 1, fig. 1).

I granuli polipantoporati (22-20-18) presentano pori forniti di membrana ed operculum microspinulato (Tav. 1, fig. 1). L'esina più spessa nelle zone mesoporiali è tectata microperforata-spinulata.

C. tomentosum si distingue da C. semidecandrum L. (De Leonardis et al. in stampa) per le maggiori dimensioni del parametro DM e del parametro Dm essendo rispettivamente nella prima $\mathrm{DM}=42 \mu \mathrm{m}$ e $\mathrm{Dm}=40 \mu \mathrm{m}$ e nella seconda $\mathrm{DM}=31 \mu \mathrm{m}$ e $\mathrm{Dm}=28 \mu \mathrm{m}$.

\section{LEGUMINOSAE}

Gillet e Crompton (1973) sulla base delle dimensioni e della morfologia pollinica propongono la chiave di identificazione di 41 taxa del Nord America. El Baba (1976) evidenzia nella struttura del reticulum l'elemento diacritico per campioni del Mediterraneo appartenenti ai subgeneri Lotoidea e Trifolium. Recentemente, Zohari ed Heller (1984), in base all'ornamentazione dell'esina, distinguono nel genere Trifolium-sette morfotipi pollinici: scrobiculato, rugulo-ruminato, reticolofoveolato, rugulato, reticolo-scrobiculato, psilato, foveato-scrobiculato.

Trifolium fragiferum ssp. bonannii mostra tre aperture composte situate nella fascia equatoriale (trizonocolporati) con colpi stretti a bordo rettilineo forniti di margo (sensu Moore e Webb 1978) e di membrana (Tav. 1, fig. 2).

L'esina di spessore costante ai poli ed all'equatore (P.es $=0,98 \mu \mathrm{m}$ e E.es $=$ $0,97 \mu \mathrm{m}$ ) è tectata soprareticolata con microperforazioni alla base dei lumina (Tav. 1, fig. 3). La sezione della parete sporodermica osservata al TEM (Tav. 1, fig. 4) evidenzia ectexina ispessita, strato columellare, foot-layer discontinuo ed endexina omogenea e spessa.

I valori dei parametri $\mathrm{P}$ ed $\mathrm{E}$ che coincidono in $T$. fragiferum ssp. bonannii $\mathrm{e}$ T. bivonae Guss. (De Leonardis et al. 1986a), assumono carattere diacritico per $\mathrm{T}$. mutabile Portenschl. $(\mathrm{P}=46(40) 36 \mu \mathrm{m}$ ed $\mathrm{E}=32(29) 27 \mu \mathrm{m}$ in De Leonardis et al. 1984a) e $T$.pratense L. ssp. semipurpureum (Strobl) Pign. $(\mathrm{P}=48(45) 41 \mu \mathrm{m}$ ed $\mathrm{E}=42(34) 26 \mu \mathrm{m}$ in De Leonardis et al. 1984c).

\section{POLYGALACEAE}

Famiglia con pollini provvisti di un numero variabile di aperture composte formate da colpi ed ora disposti nella fascia equatoriale (polizonocolporati). LobreauCallen et al. (1975) distinguono il tipo Carpolobia oligozonocolporato (7-9 aperture, raramente 10) ed il tipo Polygala polizonocolporato (20-30).

Alle aperture composte si aggiungono zone aperturali polari variamente interpretate: zone reticolate da Van Campo (1958) per P. crioptera DC.; depressioni circolari o irregolari da Moore e Webb (1978); foveolae \pm circolari da Arobba (1976) 
per $P$. flavescens DC.; lacunae da Faegri e Iversen (1975); pori da Villanueva e Ramos (1986).

Kapp (1969) ascrive il genere Polygala ai tipi stefanocolpato e stefanocolporato in base all'assenza o presenza del colpus trasversalis equatoriale (sin. di sinorato in Erdtman 1971, sinclinorato in Erdtman et al. 1961, zonorato in De Leonardis et al. 1986b).

Villanueva e Ramos (1. c.) distinguono - in base al rapporto asse polare ed equatoriale, al tipo e numero di aperture ed al perimetro equatoriale - tre morfotipi pollinici corrispondenti ai subgeneri Polygala, Chamaebutus e Brachyotropis proposti da Blake (1916) e Mcneil (1968).

I pollini di $P$. preslii - osservati dagli AA. - rientrano nel primo tipo (Tav. 3, fig. 1). Sono infatti isopolari, radiosimmetrici, breviassici; subcircolari in visione polare, con poroidi forniti di membrana granulosa e con colpi equatorialici (10-13) a margini rettilinei ed estremità arrotondate. $E^{\prime}$ inoltre visibile - in sezione ottica - il colpus trasversalis sensu Kapp (1. c.). L'esina che decresce dai poli $(2.73 \mu \mathrm{m})$ all'equatore $(2.02 \mu \mathrm{m})$ è tectata psilata.

Il valore dei parametri $\mathrm{P}$ ed $\mathrm{E}$ di $P$. preslii risulta diacritico rispetto a $P$. monspeliaca L. (De Leonardis et al. in stampa), essendo $\mathrm{P}=41(36) 33 \mu \mathrm{m}, \mathrm{E}=$ $41(38) 33 \mu \mathrm{m}$ nella prima e $\mathrm{P}=32(28) 25 \mu \mathrm{m}$ ed $\mathrm{E}=31(27) 23 \mu \mathrm{m}$ nella seconda.

\section{UMBELLIFERAE}

Erdtman (1971) definisce la famiglia stenopalinologica (2-3)- colporata, subprolata-perprolata a sexina tegillata.

Si deve a Cerceu-Larrival (1962, 1967), Cerceu-Larrival e Derouet (1975), Cerceu-Larrival e Roland-Heydacker (1978), Roland-Heydacker e Cerceu-Larrival (1978) etc. l'analisi della complessa parete sporodermica correlata ad implicazioni sistematiche e filogenetiche.

In diversi generi i pollini mostrano ipertrofia columellare per adattamento ad ambienti xerofili (Cerceu-Larrival 1965, 1971). Laserpitium garganicum ssp. siculum (Tav, 2, figg. 1-2) presenta ipertrofia polare. La sezione della parete equatoriale - osservata al TEM-mostra lo strato ectexinico con corte columellae, il foot-layer e lo spesso strato endexinico che in prossimità dell'os si ispessisce formando le costae (Tav. 2, fig. 3), mentre la sezione sporo

dermica polare presenta columellae ipertrofiche che poggiano sul foot-layer e sul sottile strato endexinico (Tav. 2, fig. 2). Pimpinella anisoides (Tav. 2, fig. 4) evidenzia ipertrofia delle columellae disposte in posizione intermedia - tra polo ed equatore- (Tav. 2, fig. 6). L'esina è rugulata in entrambi i taxa (Tav. 2, fig. 5).

$P$. anisoides differisce da $P$. peregrina L. (De Leonardis et al. 1984c) per la maggiore lunghezza dei parametri $\mathrm{P}$ ed $\mathrm{E}$, essendo nella prima $\mathrm{P}=37(33) 30 \mu \mathrm{m}$ ed $\mathrm{E}=17(14) 13 \mu \mathrm{m}$ e nella seconda $\mathrm{P}=29(26) 23 \mu \mathrm{m}$ ed $\mathrm{E}=14(12) 10 \mu \mathrm{m}$.

\section{ASTERACEAE}

La sottofamiglia delle Asteroideae è caratterizzata da quattro morfotipi pollinici (Skvarla et al. 1977). 
Nelle Senecioneae, pur predominando il tipo Senecioide, è presente il modello Helianthoide e l'Anthemoide modificato in alcuni taxa di dubbia posizione sistematica (Liabum, Sinclaria).

I granuli di Senecio aethnensis, $S$. ambiguus e $S$. lycopifolius hanno tre aperture composte -situate nella fascia equatoriale- formate da tre colpi fusiformi forniti di membrana ed ora lolongati (Tav. 3, fig. 2; Tav. 4, figg. 1, 3).

L'esina è costituita, dall'esterno verso l'interno, da quattro strati (Tav. 3 , fig. 3; Tav. 4, figg. 2, 4);

- il primo è rappresentato dal tectum perforato che supporta le spinae. In accordo alla definizione di Fraglowski (1. c.) e Praglowski e Raj (1. c.) solamente la parte solida sovratectale delle spinae può essere inclusa nella parte sculturata dell'ectexina.

- il secondo è caratterizzato dalla presenza di sottili columellae intratectali con la parte distale parzialmente fusa in modo da formare il tectum perforato e la parte basale separata dal foot-layer da uno spazio denominato cavea. In un granulo esistono tre caveae mesocolpiali, essendo i diversi strati dell'esina aderenti ai margini delle aperture (Tav. 4, fig. 4);

- il terzo strato o foot-layer privo di foramina si presenta distinto in due substrati (superiore ed inferiore). L'ipotesi del "foot-layer biforcato" attualmente basata su similarità di morfologia, densità e colorazione fra i due strati, potrebbe essere avvalorata da una indagine morfogenetica sulla sequenza di deposizione degli strati dello sporoderma.

- il quarto, endexina, è più spesso del sovrastante foot-layer.

Il valori dei parametri $\mathrm{P}$ ed $\mathrm{E}$ in $S$. aethnensis e in $S$. ambiguus (rispettivamente $\mathrm{P}=25(27) 22 \mu \mathrm{m}$ ed $\mathrm{E}=23(22) 19 \mu \mathrm{m}$ nella prima e $\mathrm{P}=30(27) 21 \mu \mathrm{m}$ ed $\mathrm{E}=30(27) 22 \mu \mathrm{m}$ nella seconda) assumono valore diacritico confermando la posizione sistematica delle due specie: Sez. Jacobeae per S. aethnensis e Sez. Incanae per $S$. ambiguus.

Le dimensioni dei pollini di $S$. lycopifolius $-\mathrm{P}=32(29) 24 \mu \mathrm{m}$ ed $=\mathrm{E}=$ $31(29) 26 \mu \mathrm{m}$ - lo accostano ai valori di $S$. ambiguus.

\section{B I BL IO G R A F I A}

ACCORSI, C.A., BANDINI MAZZANTI, M., FORLANI, L., ROSSITTO, M. -1984Palynological Italian Flora. Species of Sicilian Flora: Cards Nos. 92-99. Webbia 38:545 576.

AIELLO, M., DE LEONARDIS, W., PICCIONE, V. -1983- Palcomp. Programma per l'elaborazione di schede palinologiche. In. C.A. Accorsi et al.: Flora Palinologica Italiana. Schede elaborate tramite computer. Arch. Bot. Biogeogr. Ital. 59(1-2):57-74.

AIELLO, M., DE LEONARDIS, W., PICCIONE, V. -1984-Guida all'uso del pakage Palcomp (rel. 4.3) per la costruzione automatica di palinoschede. Boll. Acc. Gioenia Sci. Nat. 18(32.5):61-83.

AROBBA, D. -1976- Schede per una Flora Palinologica Italiana. Contributo n.3. Raccolte della costa Tirrenica. Arch. Bot. Biogeogr. Ital. 57(1-2):18-28.

BERTOLANI MARCHETTI, D., ACCORSI, C.A., AROBBA, D., BANDINI MAZZANTI, M., BERTOLANI, M., BIONDI, E., BRAGGIO, G., CIUFFI, C., DE CUNZO, T., DELlA RAGIONE, A., FORLANI, L., GUIDO, M.A., LOLLI, F., MONTANARI, C., PAOLI, P., RAIMONDO, F.M., ROSSITTO, M., TREVISAN, G. -1984- Recherches paleobotaniques sur les monts Madonie. Webbia 38:329-348. 
BLAKE, S.F. -1916- A revision of the genus Polygala in Mexico, Central America and the West Indies. Contr. Gray Herb. sér. 2,47(2): 1-122.

CERCEU-LARRIVAL, M. TH. -1962- Palynologia madagassica et mascarenica. Fam. 157. Umbelliferae. Pollen et Spores 9:431-438.

CERCEU-LARRIVAL, M.TH. -1965- Le pollen d'Ombellifères méditerranéennes, III. Scandicinae Drude. IV. Dauceae Drude. Pollen et Spores 7:35-62.

CERCEU-LARRIVAL, M.TH. -1967- Corrélations de caractères chez les grains de pollen d'Ombellifères. Rev. Paleobot. Palynol. 4:311-324.

CERCEU-LARRIVAL, M.TH. -1971- Morphologie pollinique et corrélations phylogénétiques chez les Ombelliferes. - In: The Biology and Chemistry of the Umbelliferae (ed. V.H. Heywood). Bot. J. Linn. Soc. 64, Suppl. 1:109-156.

CERCEU-LARRIVAL, M.TH., DEROUET, L. -1975- Apport de la microscopie électronique a balayage à connaissance de l'ultrastructure de l'exine de pollens d'Ombellifères. Soc. Bot. Fr. Coll. Palynologie, 122:93-95.

CERCEU-LARRIVAL, M.TH., ROLAND-HEYDACKER, F. -1978- Apport de la palynologie a la connaissance des Ombellifères actuelles et fossiles. In; Actes $2 e$ Symp. Int. Ombellifères Perpignan.

DE LEONARDIS, W., DURO, A., LONGHITANO, N., PICCIONE, V., SCALIA, C., ZIZZA, A. -1984c- Schede melissopalinologiche della Flora Apistica Siciliana. II. Boll. Acc. Gioenia Sci. Nat. 17(324): 291-375.

DE LEONARDIS, W., DURO, A., LONGHITANO, N., PICCIONE, V., SCALIA, C., ZIZZA, A. (in stampa). Schede melissopalinologiche della Fiora Apistica Siciliana. VII. Boll. Acc. Gioenia Sci. Nat. Catania.

DE LEONARDIS, W., DURO, A., PICCIONE, V., ROSSITO, M. -1984a- Flora Palinologica Italiana. Palinoschede di specie endemiche e subendemiche siciliane. Boll. Acc. Gioenia Sci. Nat. 17(324):495-527.

DE LEONARDIS, W., LONGHITANO, N., MELI, R., PICCIONE, V., ZIZZA, A. -1982Schede melissopalinologiche della Flora Apistica Siciliana. $I^{2}$. Inform. Bot. Ital. 14(1):27-93.

DE LEONARDIS, W., MARENZI, A., NAPOLEONE, I., PICCIONE, V. -1985- Banca Dati Palinologici (BA. DA. P.). Problematica e descrittori. Boll. Acc. Gioenia Sci. Nat. 18(325):5-38.

DE LEONARDIS, W., PALMIERI MATARESE, R., PICCIONE, V., ZIZZA, A. -1986aMorfobiometria pollinica all'O.M., al S.E.M. e al T.E.M. di specie endemiche siciliane. Boll. Acc. Gioenia Sci. Nat. 19(328):143-167.

DE LEONARDIS, W., PICCIONE, V., ZIZZA, A. -1984b- Premiere contribution à l'étude taxonomique du genre "Centaurea" de la Sicile sur la base des donnés polliniques, traitées par l'analyse informatique. Webbia 38:185-208.

DE LEONARDIS, W., PICCIONE, V., ZIZZA, A., SANTORO, M. -1986b- Flora Palinologica Italiana: Atlante - Glossario. Boll. Acc. Gioenia Sci. Nat. 19(329):5-82.

EL BABA, J. -1976- Contribution a l'étude cytotaxinomique et palynologique des Trifolium de la grande Mediterranée orientale. Rev. Biol. Ecol. Médit. 3(2):23-40.

ERDTMAN, G. -1960- The acetolysis metod. Sven. Bot. Tidskr. 54:561-564.

ERDTMAN, G. -1971- Pollen and Spore morphology. Plant taxonomy. Iº. Angiospermae. New York.

ERDTMAN, G., BERGLUND, B., PRAGLOWSKI, J: -1961- An introduction to a Scandinavian Pollen Flora. I ${ }^{\circ}$. Upsala.

FAEGRI, K., IVERSEN, J. -1975- Textbook of pollen analysis. 3rd. ed. Munksgaard Copenhagen.

GILLET, J.M., CROMPTON, C.W. -1973- Pollen morphology and its relationship to the taxonomy of North American Trifolium species. Pollen et Spores XV(1):91-108.

HIDEUX, M., FERGUSON, I.K. -1976- The stereostructure of the exine and its evolutionary significance in Saxifragacées sensu lato. In: I.K. Ferguson and J. Muller. The evolutionary significance of the exine. Academic Press. London and New York.

KAPP, R.O. -1969- How to know pollen and spores. W.C. Brown Co. Dubuque Iowa.

LOBREAU-CALLEN, D., OLTMANN, O., STRAKA, H. -1975- Palynologia Madagassica et Mascarenica. Fam. 99-110. Pollen et Spores 17(1):11-57. 
MCNEILL, J. -1968- Polygala. In: T.G. Tutin \& al. (Eds.). Flora Europaea 2:231-236. Cambridge.

MOLLENHAUER, H.H. -1964- Plastic embedding mixture for use in electron microscopy. Stain Technol. 39:111-114.

MOORE, P.D., WEBB, J.A. -1978- An illustrated guide to pollen analysis. Hodder and Stoughton. London.

NOWICKE, J.W. -1975- Pollen morphology in the order Centrospermae. Grana, 15:51-77.

PRAGLOWSKI, J. -1975- Importance de la mise au point des termes "structure" et "sculture" de l'exine. Soc. Bot. Fr. Coll. Palynologie 122:75-78.

PRAGLOWSKY, J., RAJ, B. -1979- On some pollen morphological conceptes. Grana 18:109-113.

REYNOLDS, E.S. -1963- The use of lead citrate at high ph as electron opaque stain electron microscopy. J. Cell. Biol. 17:208-212.

ROLAND-HEYDACKER, F., CERCEU-LARRIVAL, M.TH. -1978- Ultrastructure du tectum de pollen d'Ombellifères. Grana 17:81-89.

SKWARLA, J.J., TURNER, B.L., PATEL, V.C., TOMB, A.S. -1977-Pollen morphology in the Compositae and in morphologically related families. Academic Press. London.

VAN CAMPO, M. -1958- Palynologie Africaine. II. Bulletin de L'I.F A.N. T. XX sér. A (3):753-759.

VILLANUEVA, E., RAMOS, A. -1986- Contribucion al estudio polínico de Polygala L.L. (Polygalaceae) en la Península Ibérica. Anales Jard. Bot. Madrid 42(2):377-388.

ZOHARY, M., HELLER, D. -1984- The genus Trifolium. Jerusalem.

(Aceptado para su publicación el 15 de enero de 1989)

Dirección de los autores: Istituto e Orto Botanico . Universitá di Catania. Via Longo, 19. 95125 Catania. Italia. 

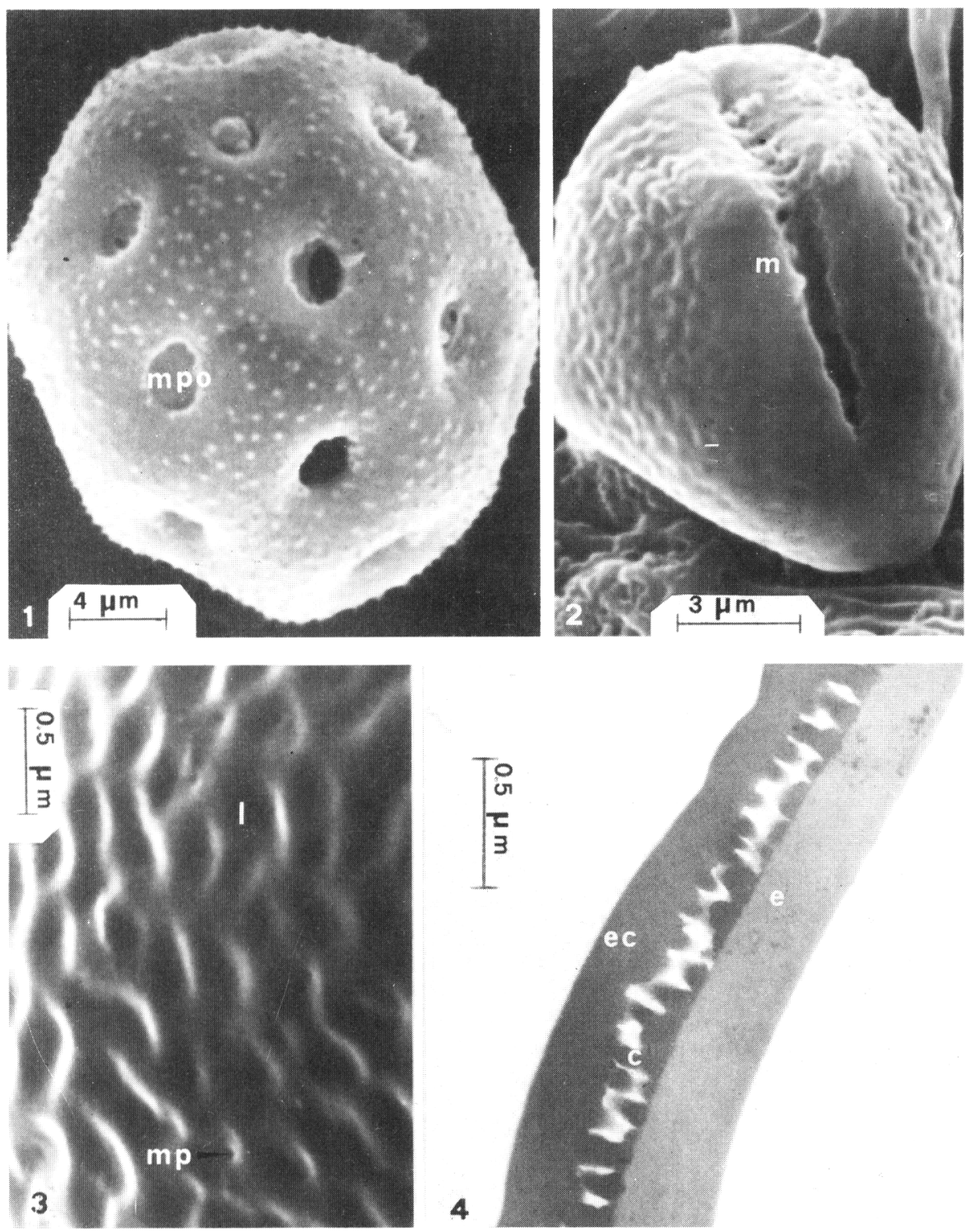

Tav. 1: 1, Cerastium tomentosum, granulo apolare. 2, Trifolium fragiferum ssp. bonannii, visione equatoriale-polare. $3-4, T$. fragiferum ssp. bonannii, sopra reticulum e structura esina. 

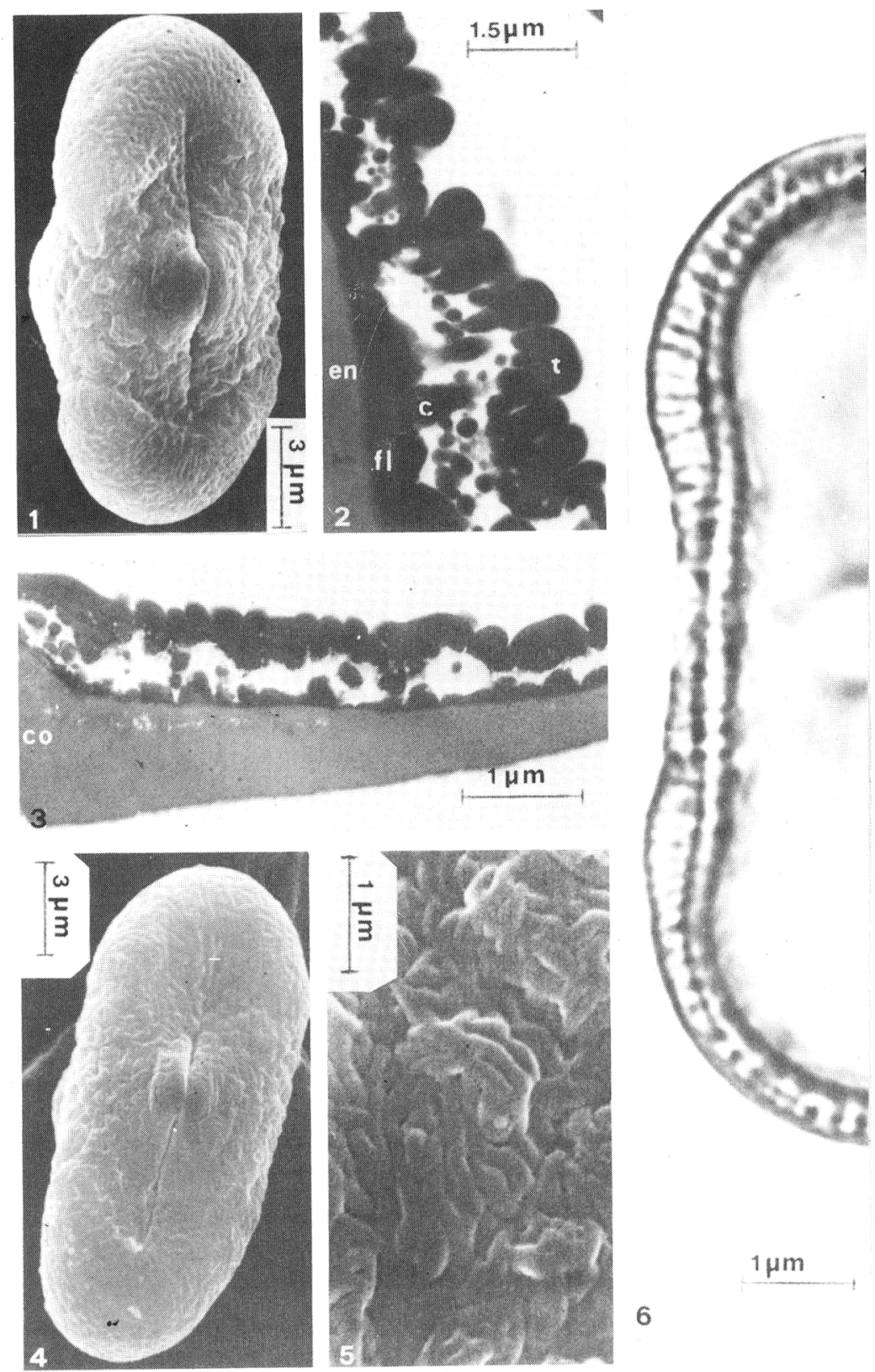

Tav. 2: 1, Laserpitium garganicum ssp. siculum, visione equatoriale. 2-3, L. garganicum ssp. siculum, ipertrofia columellare polare e struttura esina equatoriale. 4, Pimpinella anisoides, visione equatorialle. 5-6, $P$. anisoides, esina rugulata e ipertrofia columellare intermedia. 

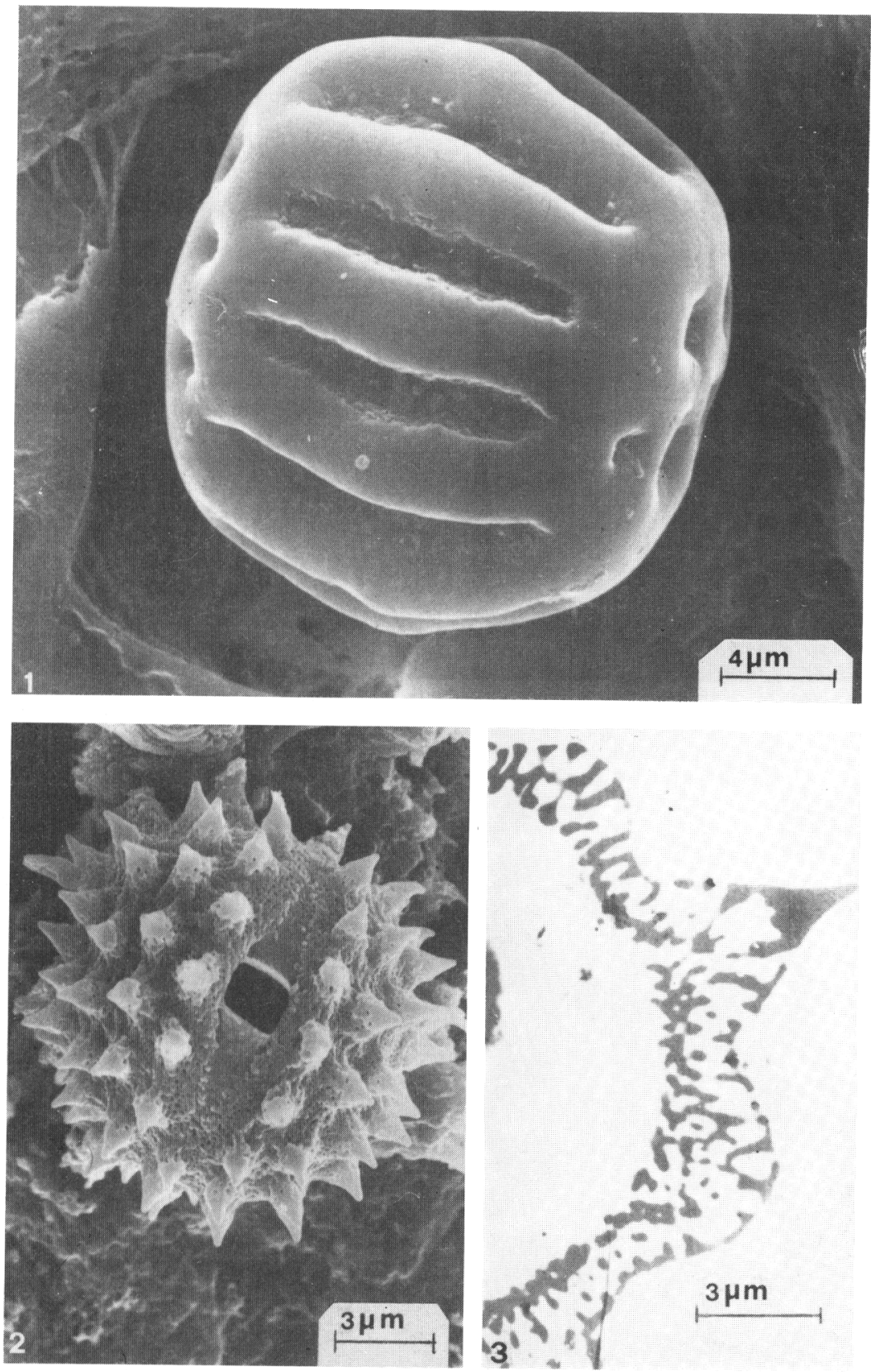

Tav. 3: 1, Polygala preslii, visione equatoriale. 2, Senecio aethnensis, visione equatoriale. 3 , $S$. aethnensis, struttura esina. 

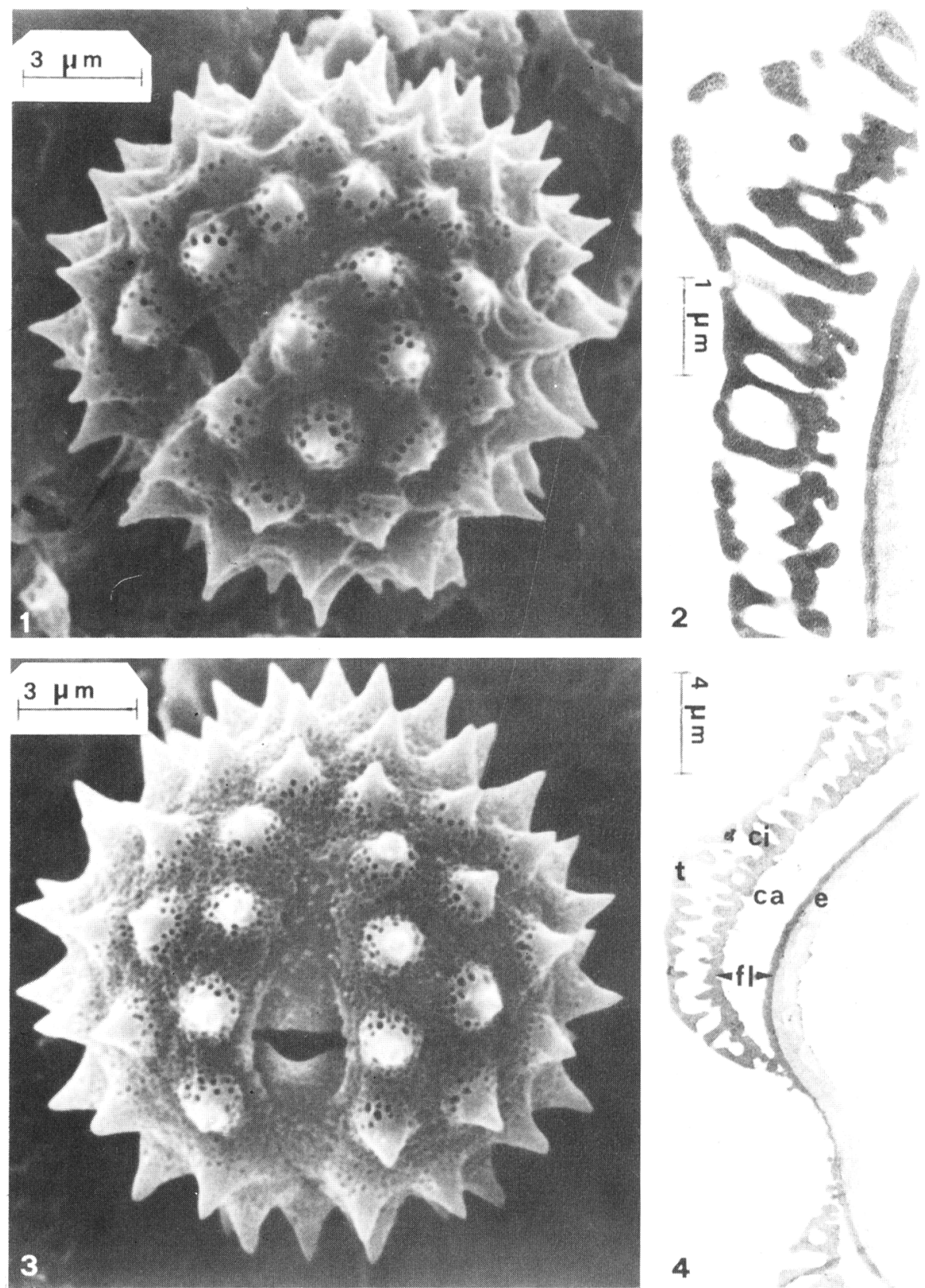

Tav. 4: 1, Senecio ambiguus, visione equatoriale. 2, S. ambiguus, struttura esina. 3, S. lycopifolius, visione equatoriale. 4, S. lycopifolius, struttura esina. 